\title{
Oral prophylaxis as an adjunct to systemic H.pylori eradication therapy
}

\author{
Pradeep P. S ${ }^{1, *}$, Manoj M. G ${ }^{2}$, Ganesh Prasad B ${ }^{3}$, Anoop M⿻ ${ }^{4}$, Asal Irfan P5 \\ ${ }^{\mathbf{1}}$ Associate Professor, ${ }^{\mathbf{2}}$ Consultant, ${ }^{\mathbf{3}}$ Reader, ${ }^{4}$ Senior Resident, ${ }^{\mathbf{5}}$ Junior Resident, ${ }^{\mathbf{1 , 4}, \mathbf{5}}$ Dept. of Dentistry, ${ }^{\mathbf{2}}$ Dept. of Gastroenterology, ${ }^{\mathbf{3}}$ Dept. of \\ Oral Pathology, ${ }^{\mathbf{1}, \mathbf{2}, \mathbf{4}, \mathbf{5}}$ DM Wayanad Institute of Medical Sciences, Kerala, AJ Institute of Dental Sciences, Mangalore, Karnataka, India \\ *Corresponding Author: Pradeep P. S \\ Email: drpradeep.paisari@gmail.com
}

\begin{abstract}
Introduction: Helicobacter pylori is the known bacteria, causing multiple gastric disorders. Its prevalence in dental plaque noted by multiple researchers. The presence of helicobacter pylori in dental plaque even after systemic eradication done using triple drug therapy noted by few. Purpose of this study was to establish need of oral prophylaxis as an adjunct to systemic eradication of H.pylori.

Materials and Methods: Total of 22 H.pylori positive patients divided in two groups irrespective of gender. 14 patient underwent oral prophylaxis and remaining 8 subjects did not, along with triple drug therapy for systemic eradication of H.pylori. Plaque samples from only posteroior teeth collected and PCR was done for detection of H.pylori strains after 6 months.

Results: Statistical significant reduction in prevalence of H.pylori noted in patients undergone oral prophylaxis along with anti-H pylori therapy.

Conclusion: Oral prophylaxis has to be considered along with anti-H pylori eradication therapy to prevent dental plaque acting as a reservoir causing reinfection.
\end{abstract}

Keywords: Oral prophylaxis, Helicobacter pylori, Reservoir, Systemic eradication.

\section{Introduction}

Helicobacter pylori has been known as etiological gram-negative, curved microaerophilic organism of gastritis, gastric and duodenal ulcer formation, gastric mucosaassociated lymphoid tissue lymphoma and gastric carcinoma. ${ }^{1,2}$ While over $50 \%$ of the worlds population caries this infection, atleast $10 \%$ of individuals are effected by gastritis or gastric ulcer during their lifetime. ${ }^{3}$ First successful isolation and culture of a spiral bacterial species, later known as Helicobacter pylori from human stomach was done by Barry and Robin Warren. ${ }^{4}$ Marshall and Morris performed self ingestion experiments and later with volunteers, demonstrated that these bacteria can colonize the human stomach, there by inducing inflammation of the gastric mucosa. ${ }^{5,6}$ These initial data strongly stimulated further research, which showed that gastric colonization with H.pylori lead to variety of disorders mentioned above. Publications on research related to H.pylori has rapidly increased, from few in early 1990 to approximately more than 1,500 in recent years (www.pubmed.gov). Developed countries observed decrease in the incidence of gastric carcinoma parallels the decreasing rate of the prevalence of H.pylori infection in recent years. ${ }^{7,8}$ Even though the prevalence of H.pylori in the western world is decreasing, it remains widespread in developing and underdeveloped countries. Despite of high attention to this important issue, transmission of H.pylori still not understood clearly. Although transmission mode of H.pylori not fully understood as infection in humans appears to be involving the oral route. At the same time oral cavity may act as possible extra gastric reservoir for $\mathrm{H}$ pylori, which may lead to recurrent infection even after systemic eradication. ${ }^{9}$ Saliva, microbiota from the dorsum of the tongue, dental plaque, oral ulceration and oral neoplasias are tested positive for H.pylori in various studies. ${ }^{10-12}$ Infection with
H.pylori can be diagnosed by variety of tests and successfully treated with systemic antibiotic therapy or famously called as HP kit (Cap. Amoxicillin / Clarithromycin and one PPI for the period of two weeks). Current treatment modalities has been successful in management of H.pylori positive gastritis, but the recurrence rate is high. ${ }^{10}$ Antibiotic resistance already started effecting the treatment outcome and preventive vaccination strategies still do no exist inspite of impact of this bacteria.

Purpose of this study was to evaluate effectiveness of oral prophylaxis as adjunct to systemic H.pylori eradication therapy in recurrence of H.pylori prevalence in dental plaque.

\section{Materials and Methods}

Total of 22 patients undergoing systemic eradication of H.pylori using HP kit from Department of gastroenterology included in this study between October 2016 till March 2017. Patients were divided in two groups. Group I consisted of patients who were willing to undergo oral prophylaxis on the day of starting of systemic antibiotics. Group II consisted of patients who are not willing for the oral prophylaxis. Follow up of these patients were done for the period of six months till September 2017. At the end of second year subgingical plaque was collected from all samples and sent for PCR for detection of H.pylori. Reinfection of subjects during this period also documented.

\section{Inclusion Criteria}

1. Patients above 30 years of age irrespective of sex

2. Dentate patients only

3. Chronic generalised periodontitis patients with tested positive for H.pylori from Department of Gastoenterology by antral biopsy 


\section{Exclusion Criteria}

1. Patients with substance dependence (Smoking, Alcohol etc)

2. Patients with any kind of systemic diseases other than gastritis

3. Patients who had taken PPI, Bismuth containing compounds or antibiotics in last two months.

Management of Group I Patients: Patients who were willing for subgingival scaling and root planning were included in this study. On the first day of beginning of the HP kit therapy patients were treated with oral prophylaxis. Chlorhexidine Gluconate $0.2 \%$ mouth wash was prescribed for a week. No additional medication was given neither systemically nor for topical application.

Management of Group II Patients: Patients not willing for oral prophylaxis were included in this group. Details of this patients recorded for documentation purpose.

Collection of sample and PCR: Sterile curette was used for careful removal of subgingival plaque from posterior teeth only. This was done for both the group in the month of January 2018. Sample was carried to the institution laboratory using buffered saline for PCR. Polymerase chain reaction (PCR) is molecular biology technique for enzymatically replicating DNA without using living organism. It allows a small amount of DNA to be amplified exponentially.

H.pylori positive samples in both the groups noted. Statistical analysis done for H.pylori positive sample in patients undergone oral prophylaxis with patients who did not wish for oral prophylaxis along with anti-H pylori therapy. Written consent from all subjects obtained and this study was approved by internal ethics committee on human studies of the institution.

\section{Results}

This study consisted of two groups. Group I consisted of 14 patients. Mean age of these patients was 38.6 years and had 9 males and 5 female patients. Group II consisted total of 8 patients in which 6 were males and 2 females with mean age of 47.7 years (Table 1 ).

Table 1: Distribution of study subjects according to age

\begin{tabular}{|l|c|c|c|c|}
\hline \multicolumn{1}{|c|}{ Groups } & $\mathbf{N}$ & Mean & $\begin{array}{c}\text { Std. } \\
\text { Deviation }\end{array}$ & $\begin{array}{c}\text { Std. Error } \\
\text { Mean }\end{array}$ \\
\hline Group I & 14 & 38.6429 & 4.23551 & 1.13199 \\
\hline Group II & 8 & 47.7500 & 7.10634 & 2.51247 \\
\hline
\end{tabular}

Prevalence of H.pylori tested from dental plaque of posterior teeth from all subjects of this study. In Group I three patients tested positive for H.pylori after 6 months of systemic eradication and oral prophylaxis. All 8 patients from Group II tested positive for H.pyloi after 6 months of systemic eradication only. This values were highly significant statistically. (Table 2)

Table 2: Comparison of $\mathrm{H}$ pylori among groups

\begin{tabular}{|l|c|c|c|c|c|}
\hline Groups & $\mathbf{N}$ & Mean Rank & Sum of Ranks & Mann Whitney U test & Pp value and Significance \\
\hline Group I & 14 & 14.64 & 205.00 & \multirow{2}{*}{12.00} & \multirow{2}{*}{0.001 Highly Significant } \\
\hline Group II & 8 & 6.00 & 48.00 & & \\
\hline
\end{tabular}

\begin{tabular}{|c|c|c|c|c|}
\hline & & & \multirow[t]{2}{*}{ Total } \\
\hline & & Positive & Negative & \\
\hline \multirow[t]{2}{*}{ Groups } & Group I & $\begin{array}{c}3 \\
27.3 \%\end{array}$ & $\begin{array}{c}11 \\
100.0 \%\end{array}$ & $\begin{array}{c}14 \\
63.6 \%\end{array}$ \\
\hline & Group II & $\begin{array}{c}8 \\
72.7 \%\end{array}$ & $\begin{array}{c}0 \\
.0 \% \\
\end{array}$ & $\begin{array}{c}8 \\
36.4 \% \\
\end{array}$ \\
\hline
\end{tabular}

\section{$\chi^{2}=12.571, p=0.001$ Highly Significant}

\section{Discussion}

Significance of H.pylori is undisputed due to high infection rate of the world's population. Severe outcome of H.pylori infection in humans, mode of transmission is still a major concern. Dental plaque is a known reservoir of H.pylori by many researchers. ${ }^{13}$ Wide range of $1 \%$ to $82.1 \%$ prevalence rate of coinfection among the respective study population reported by different investigators. Difference in diagnostic tools employed by researchers may be partly responsible for this wide variation. H.pylori detection using urease tests reported high prevalence rates in dental plaque. Among the studies which used urease test, one test reported a $25.2 \%$ prevalence of coinfection, all other studies reporting coinfection rate excess of $32 \%$, with only one study reporting $81.3 \% .^{14}$ More reliable PCR involving studies, the prevalence rates have ranged between $0 \%$ and $47.6 \%$ with only 5 in 11 studies reporting prevalence rate above $30 \%{ }^{15}$ When Microbial culture was employed to detect H.pylori, low rates of prevalence of coinfection reported. ${ }^{16,17}$ The studies reviewed which used immunoassays for detection of this bacteria in dental plaque samples, the prevalence rates reported were $23.6 \%$ and $47.6 \% .^{18}$

Possibility of H.pylori inducing periodontal or gingival infections also been studied by many. Some studies have reported relationship between periodontitis and H.pylori positively..$^{19}$ Exactly the opposite also has been showed in some studies which states no association between H.pylori and periodontal disease. ${ }^{20}$ Dye et al performed a large scale epidemiological study which evaluated the relationship between H.pylori and abnormal periodontal conditions ${ }^{19}$. 4504 subjects irrespective of sex of aged 20 to 59 years who 
completed a periodontal examination and tested positive for H.pylori antibodies were examined. Pocket depth of $5 \mathrm{~mm}$ or more were found with increased odds of H.pylori seropositively. These findings concluded that poor periodontal health with advanced periodontal pockets may be associated with H.pylori. P. S Anand et al conducted a case control study to find out association between periodontal disease and H.pylori infection in 134 dyspectic patients. ${ }^{21}$ Periodontal status determined patient as healthy or diseased when patients were chosen from those seeking upper gastrointerstinal endoscopy. Among the subjects, 30 patients out of 65 had periodontal disease compared to only 20 out of 69 in comparison to the controls. In a study conducted by Berroteran et al on venezuelan population failed to establish a relation between periodontal infection and periodontal disease. ${ }^{22}$ In 32 dyspeptic patients and 20 asymptomatic subjects gingival and plaque indices were used to assess the gingival and oral hygiene status. It was concluded in this study that patients with poor oral hygiene and periodontal status had similar prevalence of H.pylori to patients with good oral hygiene.

Butt et al treated 27 out of 82 Helicobacter pylori positive patients with anti-H therapy (triple therapy-2 antibiotics and 1 proton pump inhibitor) alone and reported $100 \%$ prevalence of H.pylori in dental plaque of these patients after 10 days. $^{23}$ In another study 75 H.pylori positive, dyspeptic patients were treated with anti-H pylori therapy. ${ }^{24}$ This study $91 \%$ patients had tested positive for H.pylori in dental plaque were as $87 \%$ positive for gastric mucosa. After one month of Anti-H therapy in all these patients, when procedures are repeated tested positive for H.pylori in dental plaque. Gao et al employed PCR for pathogen detection, they reported that among 37 patients prevalence of H.pylori colonization was $29.7 \%$ and $43.2 \%$ respectively, at 4 weeks and 1 year after anti-H therapy. ${ }^{25}$ In a published study it is noted that H.pylori persisted in the dental plaque of $92.9 \%$ of patients after anti-H therapy. ${ }^{26}$

In a study similar to this study Zaric et al noted that, in 22 patients who received both anti-H pylori therapy and periodontal therapy, only 6 patients tested positive of H.pylori after 3 months of completion of therapy. ${ }^{27}$ In the same study $66.7 \%$ patients tested positive for H.pylori after three months of anti-H therapy in the dental plaque without dental treatment. Nonsurgical periodontal therapy effecting on H.pylori prevalence after anti-H therapy discussed by Butt et al. ${ }^{23}$ This study categorized 82 patients in three groups. In the group which received only anti-H pylori therapy treated $100 \%$ prevalence of H.pylori in dental plaque. In the group which received periodontal therapy along with anti-H pylori therapy resulted in only $16 \%$ patients being positive for H.pylori in the dental plaque. Combination of anti-H pylori therapy (triple therapy) and periodontal therapy studied by Gao et al. ${ }^{25}$ This study treated 37 patients with anti-H therapy alone and 43 patients combination with periodontal therapy. The detection rates of H.pylori in the dental plaque for both the groups at four weeks after intervention were $29.7 \%$ and $4.7 \%$ were as $43.2 \%$ and $18.6 \%$ after one year respectively. Findings of this study is similar to most of the studies mentioned above which supports the role of oral prophylaxis in systemic eradication of helicobacter pylori.

\section{Conclusion}

The role of Helicobater pylori in development of many gastric diseases is proven in literature. Prevalence of helicobacter pylori in deep periodontal pockets tested positive in multiple studies. As proven in this study, many other studies also showed combination of periodontal therapy along with systemic eradication of helicobacter pylori gives better clinical results when compared to anti-H pylori treatment alone. Even though high prevalence of H.pylori noted in dental plaque even after systemic eradication is a proven fact, its difficult to draw conclusions on reinfection due to prevalence in dental plaque. Further controlled randomized clinical trials are necessary to establish role of H.pylori in dental plaque causing reinfection. However authors of this study strongly recommend multidisciplinary approach in management of systemic eradication of H.pylori by adding periodontal therapy.

\section{Conflict of Interest: Nil.}

\section{References}

1. Kilmartin CM. Dental implications of helicobacter pylori. $J$ Can Dent Assoc. 2002;68:489-493.

2. Martinez-Gomis J, Diouf A, Lakhssassi N, Sixou M, Absence of helicobacter pylori in the oral cavity of 10 non-dyspeptic subjects demonstrated by real time polymerase chain reaction. Oral Microbiol Immunol. 2006;21:407-410.

3. Dowsett SA, Kowolik MJ. Oral helicobacter pylori. Can we stomach it? Crit Rev Oral Biol Med. 2003;14:226-233.

4. Warren J.R, Marshall B.J. Unidentified curved bacilli on gastric epithelium in active chronic gastitis. Lancet $i$. 1983;1273-1275.

5. Marshall B.J, J.A. Armstrong, D.B. M. McGechie, and R.J.Glancy. Attempt to fulfil Koch's postulates for puloric campylobacter. Med J Austr. 1985;142:436-439.

6. Morris. A, And G. Nicholson. Ingestion of Campylobater pyloridis cause gastritis and raised fasting gastric $\mathrm{pH}$. 1987;82:192-199.

7. Parasonnet J. The incident of Helicobater pylori infection. Aliment Pharmacol Ther.1995;9:45-51.

8. Howson CP, Hiyama T, Wynder EL. The decline in gastric cancer: epidemiology of an unplanned triumph. Epidemiol Rev.1986;8:1-27.

9. Miyabayashi H, Furihata K, Shimizu T, Ueno I, Akamatsu T. Influence of oral Helicobacter pylori on the success of eradication therapy against Helicobacter pylori. Helicobacter. 2000;5:30-37.

10. Gebara EC, Faria CM, Pannuti C, Chehter L, Mayer MP, Lapa L. Persistence of helicobater pylori in oral cavity after systemic eradication therapy. J Clin Periodontol. 2006;33:329-33.

11. Kignel S, Pina FA, Andre EA, Mayer MP, Birman EG. Occurance of helicobater pylori in dental plaque and saliva of dyspeptic patients. Oral Dis. 2005;11:17-21.

12. Krajden S, Fuksa M, Anderson J, Kempston J, Aoccia A, Petrea C, et al. Examination of human stomach biopsies, saliva and dental plaque for campylobacter pylori. J Clin Microbiol.1982;27:1397-1398. 
13. A. Al-Ahmad, A. Kurschner, S. Weckesser, A. Wittmer, H. Rauberger, T. Jakob, E. Hellwig, M. Kist, B. Waidner. Is Helicobater pylori resident or transient in the human oral cavity?

14. S. Agarwal and K.D Jithendra. Presence of helicobacter pylori in subgingival plaque of periodontitis patients with and without dyspepsia detected by polymerase chain reaction and culture. $J$ Indian Soc Periodontol. 2012;16(3):398-403.

15. P. Majmudar, S.M. Shah, K.R. Dhunjibhoy, H.G. Desai. Isolation of helicobacter pylori from dental plaques in healthy volunteers. Indian J Gastroenterol. 1990;9(4):271-272.

16. A. Oshowo, D. Gillam, A. Botha. Helicobater pylori: the mouth, stomach, and gut axis. Ann Periodontol. 1998;3(1):276-280.

17. M. Czesnikiewicz-Guzik, E. Karczewska, W. Bielanski. Association of the presence of helicobacter pylori in the oral cavity and in the stomach. $J$ Physiol Pharmacol. 2004;55(2):105-115.

18. L. Checchi, P. Felice, C. Acciardi. Absence of helicobater pylori in dental plaque assessed by stool test. $\mathrm{Am} \mathrm{J}$ Gastroenterol. 2000;95(10):3005-3006.

19. M. Al Asqah, N. Al Hamoudi, S. Anil, A. Al Jebreen, W.K. Al-Hamoundi. Is the presence of Helicobacter pylori in the dental. Canadian J Gastroenterol. 2009;23(3):177-179.

20. M. Umeda, H. Kobayashi, Y. Takeuchi. High prevalence of Helicobacter pylori detected by PCR in oral cavities of periodontitis patients. J Periodontol. 2003;74(1):129-134.

21. P. S Anand, k. Nandakumar, K.T Shenoy. Are dental plaque, poor oral hygiene, and periodontal disease associated with
Helicobacter pylori infection?. J Periodontol. 2006;77(4):692698.

22. A. Berroteran, M. Perrone, M. Correnti. Detection of helicobater pylori DNA in the oral cavity and gastroduodenal system of a venezuelan population. J Med Microbiol. 2002;51(9).764-770.

23. A.K Butt, A.A Khan, B.A Suleman, R. Bedi. Randomized clinical trial of helicobacter pylori from the dental plaque. $\mathrm{Br} J$ Surg. 2001;88(2):206.

24. A.K. Gurbuz, M. Ozel, Y. Yazgan. M. Celik, S. Yildirim. Oral colonization of Helicobacter pylori: risk factors and response to eradication therapy. 2003;96(3):244-247.

25. J. Gao, Y. Li, Q. Wang, C. Qi, S. Zhu. Correlation between distrbution of helicobacter pylori in oral cavity and chronic stomach conditions. J Huazhong Univ Sci Technol-Med Sci. 2011;31(3):2011.

26. F.M. Suk. S.H. Chen, Y.S.Ho. It is difficult to eradicate helicobacter pylori from dental plaque by tripple therapy. Chin Med J. 2002;65(10):468-473.

27. S. Zaric, B. Bojic, L. Jankovic. Periodontal therapy improves gastric helicobacter pylori eradication. J Dent Res. 2009;88(10):946-950.

How to cite this article: Pradeep P. S, Manoj M. G, Ganesh P. B, Anoop M, Asal I. P. Oral prophylaxis as an adjunct to systemic H.pylori eradication therapy. Int J Oral Health Dent. 2018;4(4):230-233. 